\title{
Compact assembly-free vector bend sensor based on all-in-fiber-core Mach-Zehnder interferometer
}

\author{
PengCheng Chen $^{1}$, Xuewen Shu ${ }^{1}{ }^{*}$, Kate Sugden ${ }^{2}$ \\ ${ }^{I}$ Wuhan National Laboratory for Optoelectronics \& School of Optoelectronic Science and Engineering, Huazhong University of Science and \\ Technology, Wuhan 430074, China \\ ${ }^{2}$ Aston Institute of Photonic Technologies, Aston University, Birmingham B4 7ET, UK \\ *Corresponding author:xshu@hust.edu.cn
}

Received XX Month XXXX; revised XX Month, XXXX; accepted XX Month XXXX; posted XX Month XXXX (Doc. ID XXXXX); published XX Month XXXX

\begin{abstract}
A novel low-cost, compact, assembly-free and sensitive optical fiber curvature sensor is presented. This device consists of an off-axis positive refractive index modified zone (PRIMZ), induced by direct femtosecond laser, written in single mode fiber (SMF) core. The PRIMZ transforms the original SMF section into a few-mode fiber (FMF). As a result, the whole fiber forms an assembly-free "SMF-FMF-SMF" sandwich Mach-Zehnder interferometer. When the device is bent, a directiondependent spectral shift of the interference pattern is produced. The sensitivity of the sensor is up to 2.53 and $2.24 \mathrm{~nm} / \mathrm{m}^{-1}$ for the $0^{\circ}$ and $180^{\circ}$ orientations in a wide bend range (from $0-4 \mathrm{~m}^{-1}$ ). In addition, the device is immune to surrounding refractive index and has low temperature crosstalk, which make it very attractive for practical structural monitoring applications. (C) 2017 Optical Society of America
\end{abstract}

OCIS codes: (230.1150) All-optical devices; (280.4788) Optical sensing and sensors; (220.0220) Optical design and fabrication; (230.0230) Optical devices; (120.3180) Interferometry.

http://dx.doi.org/10.1364/OL.99.099999

The ability to make continuous vector bending measurements is extremely important for numerous applications in mechanical engineering, structural health monitoring and automotive industries. Optical fiber sensors are very suited to these applications due to their unique advantages of compactness, lightweight, stability, fast response, and immunity to electromagnetic interference. Various fiber-based schemes have been proposed to realize direction-sensitive bending sensors. The schemes can be divided into two main categories: grating-based and interferometer-based. In general, grating-based sensors, including fiber Bragg gratings (FBGs) and long period gratings (LPGs) vector bending sensors, can only be achieved in specifically designed fibers with axial asymmetrical structures such as Dshaped fiber [1], eccentric core fiber[2, 3], multicore fiber [4, 5], and photonic crystal fiber[6, 7], or by inscribing asymmetric refractive index gratings[8-10]. FBG-based bend sensors usually exhibit low bend sensitivity. LPGs have higher bend sensitive, but they suffer from strong surrounding medium crosstalk since they are very sensitive to surrounding refractive index and temperature. Fiber interferometers based on modal interference are widely reported to achieve a vector bend sensing with relatively high sensitivity and have been demonstrated using multicore fibers[11-13], photonic crystal fibers [14, 15] and directional lateral-offset splicing[16-18]. Unfortunately, special fibers are high cost and directional fiber lateral-offset splicing is fragile and complex. In addition, it is usually difficult to splice special fibers with standard single-mode fiber (SMF) and the fabrication of the bend sensors [11-18] involves a multi-step manual process that usually leads to low reproducibility.

In this Letter, we report a simple interferometric bend sensor based on an off-axial positive refractive index modified zone (PRIMZ) in SMF core, which in turn forms an all-in-fiber-core Mach-Zehnder interferometer (MZI). The PRIMZ is induced in a one-step direct femtosecond (fs) laser writing process. Compared with previous structures, our structure has several distinct advantages: simple fabrication; compact structure; assembly-free and low cost (since only standard SMF is required). We believe that the sensing configuration proposed here overcomes the main limitations of the majority of previously proposed optical fiber vector bend sensors.

The fabrication setup of the in-fiber-core MZI is shown in Fig. 1(a). An in-fiber core PRIMZ was induced in a section of coating stripped SMF using a fs-laser. The fabrication of this device is very straightforward and the specific fabrication equipment and process has been discussed in our previous work [19]. Figure 1(b) and (c) show a microscope image of the fabricated MZI viewed from the laser direction, where the PRIMZ can be seen clearly. 


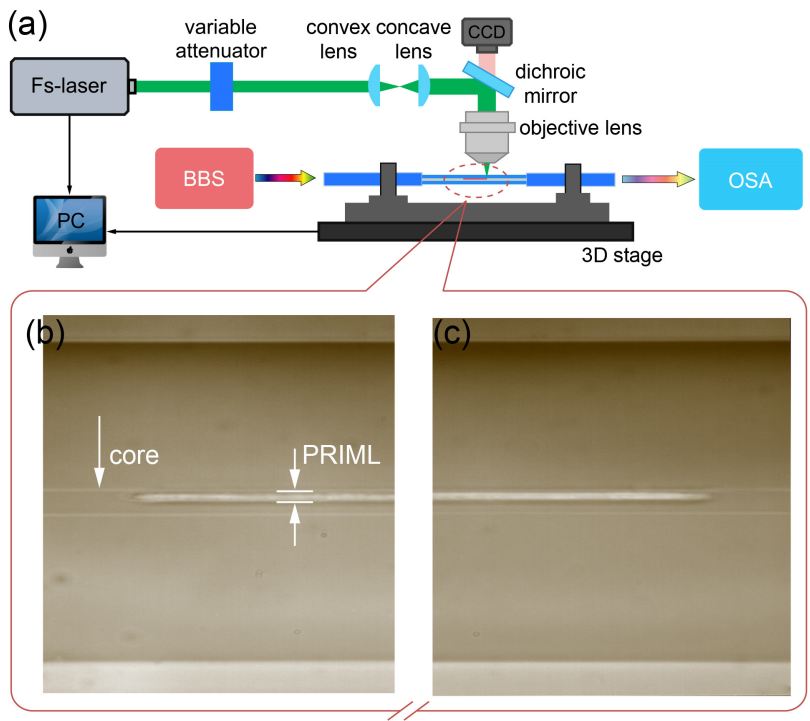

Fig. 1. (a) Schematic diagram of the proposed all-in-fiber-core MZI. (b)(c) The microscope images of the fabricated MZI near two ends.

The working mechanism of our interferometer has been discussed in detail in [19]. Basically, the fundamental mode of the SMF excites a new fundamental mode and a higher-order mode in the PRIMZ fiber section, which gives rise to an interference effect.

The transmission of our device can be expressed as:

$$
I_{T}=I_{1}+I_{2}+2 \sqrt{I_{1} \cdot I_{2}} \cos \left(2 \pi \Delta n_{\text {eff }} L / \lambda\right)
$$

In Eq. (1), $I_{1}$ and $I_{2}$ represent the intensities of the interfering modes; $\Delta n_{\text {eff }}$ is the effective index difference between the interfering modes; $L$ is the PRIMZ length, and $\lambda$ is the wavelength of the light in vacuum. Intensity dips appear when $2 \pi \Delta n L / \lambda=(2 m+1) \pi$, where $m$ is an integer, corresponding to wavelengths at:

$$
\lambda_{m}=\frac{2 \Delta n_{e f f} L}{2 m+1}
$$

When the interferometer is bent, the refractive index profile of the modified fiber will change, which will result in the change of the effective refractive indices of both the fundamental mode and highorder modes. When the $\Delta n_{\text {eff }}$ is changed, the wavelength of the interference dip will also shift.

The bending characteristics of the implemented MZI with $2000 \mu \mathrm{m}$ length have been evaluated using the experimental setup illustrated in Fig. 2(a). The sensing section was mounted between two high-precision full $360^{\circ}$ bare fiber rotation stages; the rotation stages were mounted on a pair of 1-dimension displacement stage with a resolution of $10 \mu \mathrm{m}$. The curvature $(C)$ of the sensor is given by [18]

$$
C^{-1} \sin \left(\frac{L_{0} C}{2}\right)=\frac{L}{2}
$$

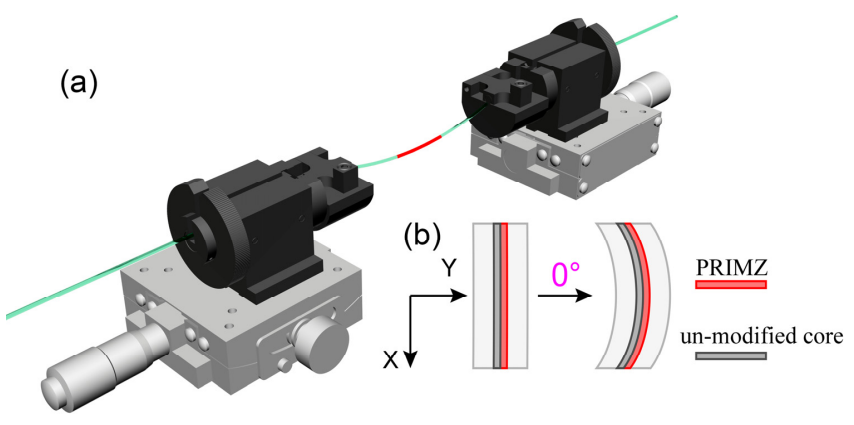

Fig. 2. (a) Setup used to characterize the bending response of the MZI. (b) Relationship between the defined orientation of $0^{\circ}$ and the fabricated PRIMZ.

Where $L_{0}$ is the initial length, $L$ is the reduced length between the two holders and $C$ is the curvature. In our experiment, $L_{0}$ is 20 $\mathrm{cm}$ and the final length $L$ is $19.471 \mathrm{~cm}$ (corresponding to $4 \mathrm{~m}^{-1}$ bending). Here, the fiber at the orientation of $0^{\circ}$ is defined, as shown in Fig. 2(b). The light from a super continuum broadband source (BBS) was passed through the device and the transmission spectra were recorded using an optical spectrum analyzer with a resolution of $0.02 \mathrm{~nm}$ (OSA, AQ6370C).

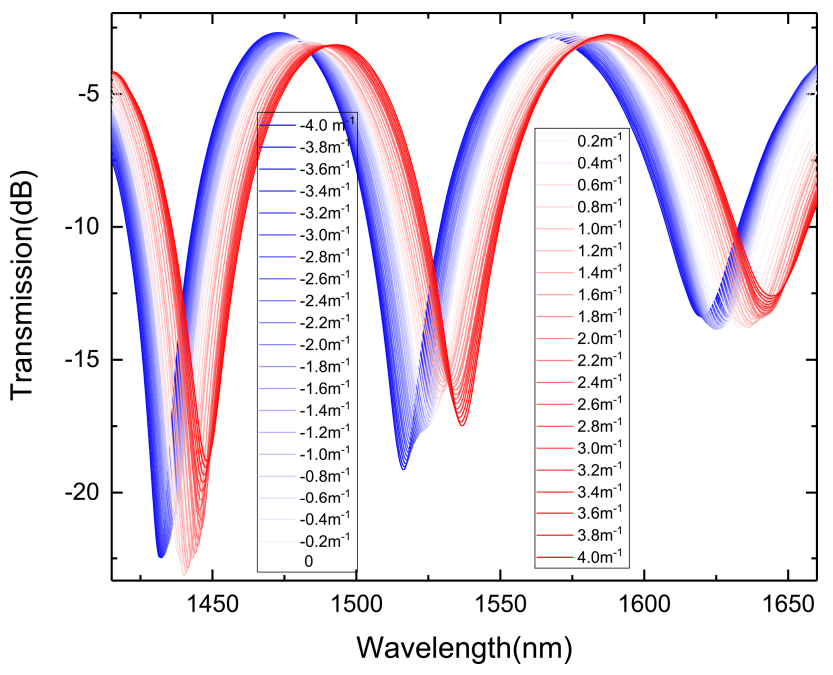

Fig. 3. Measured transmission spectra with varying curvature in the $0^{\circ}$ direction (red curve) and $180^{\circ}$ direction (blue curve).

Figure 3 shows the spectral response of the device during bending at the orientations of $0^{\circ}$ and $180^{\circ}\left(0-4 \mathrm{~m}^{-1}\right.$ bending range with step of $0.2 \mathrm{~m}^{-1}$ ). It is clear that the bending response of the allin-fiber-core MZI is strongly dependent on the bending direction. A shift to a longer wavelength (red shift) was observed when the interferometer was bent in the $0^{\circ}$ orientation and a shift to shorter wavelengths (blue shift) was observed when the device was bent in the $180^{\circ}$ direction. 

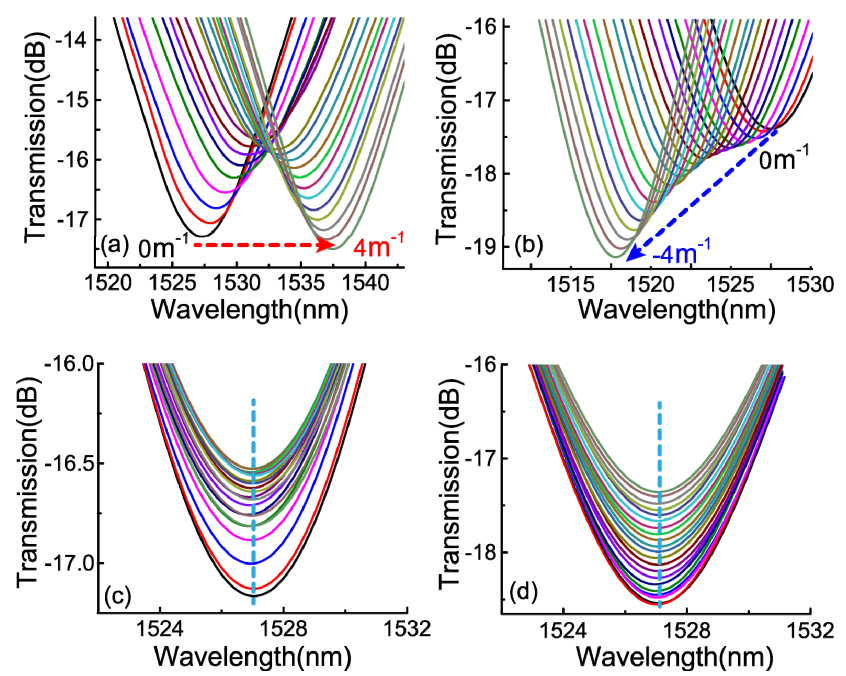

Fig. 4. Magnifying area of the transmission spectra central wavelength $\sim 1527 \mathrm{~nm}$ shift with varying curvature at the orientation of (a) $0^{\circ}$, (b) $180^{\circ}$, (c) $90^{\circ}$ and (d) $270^{\circ}$.

To observe the wavelength shift more clearly, Figs. 4(a) and 4(b) show the magnified area of the spectral response of the device during bending at the orientations of $0^{\circ}$ and $180^{\circ}$. Figures 4 (c) and 4(d) show the spectral evolution of the MZI when bending at orientations of $90^{\circ}$ and $270^{\circ}$, in which there is little change in the interference wavelengths.

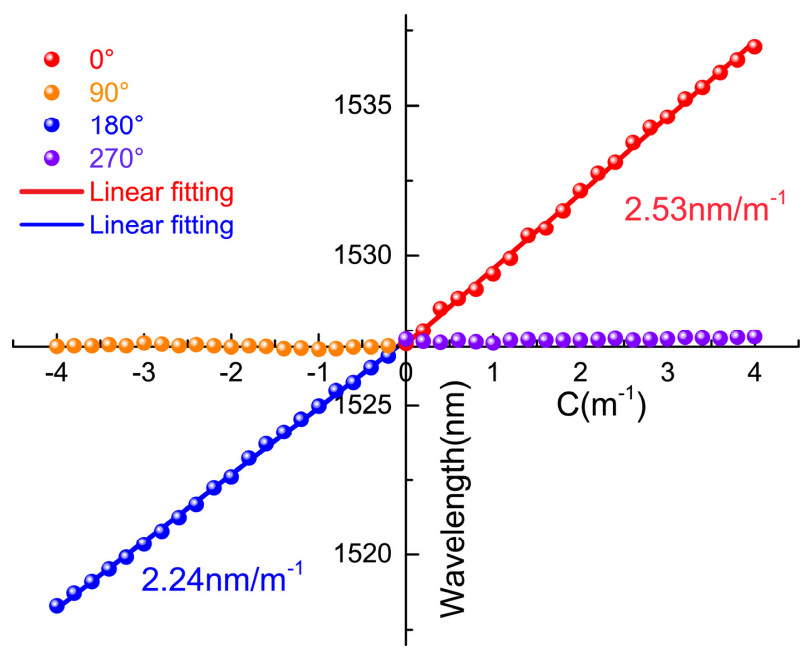

Fig. 5. Linear fits of the central wavelength $1527 \mathrm{~nm}$ against for four orientations.

Figure 5 shows the measured the interference wavelengths of the dip at around $1527 \mathrm{~nm}$ against the bending curvature for four orientations. It is evident that the bending response exhibits good linearity. The device shows the sensitivities of $2.53 \mathrm{~nm} / \mathrm{m}^{-1}$ and $2.24 \mathrm{~nm} / \mathrm{m}^{-1}$ for the $0^{\circ}$ and $180^{\circ}$ orientations, respectively. The $90^{\circ}$ and $270^{\circ}$ orientations have no obvious bending sensitivity (no wavelength change), which indicates that the $\Delta n_{\text {eff }}$ approximately keeps as a constant.

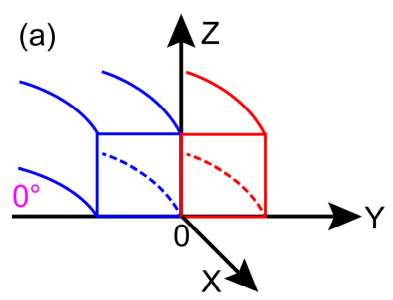

(b)

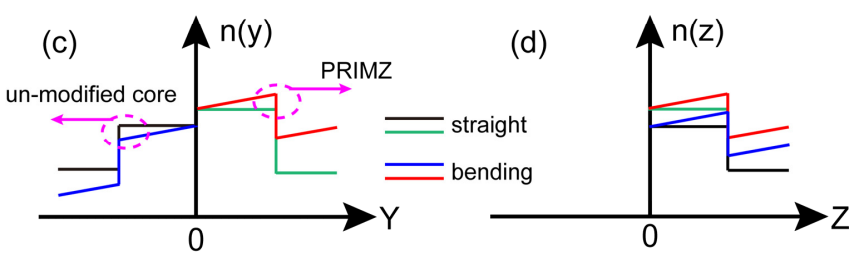

Fig. 6. (a) and (c) Geometry and its corresponding refractive index profile of bending MZI at $0^{\circ}$. (b) and (d) Geometry and its corresponding refractive index profile of bending MZI at $90^{\circ}$.

The modeling of the bending response can be determined through the use of an equivalent straight waveguide (ESW) whose refractive index profile is modified from the bent waveguide through a conformal transformation [20]

$$
n(r)=n_{0}(1+r \cdot C)
$$

Here, $n_{0}$ is the index of the straight fiber, $n(r)$ is the RI of the bending fiber, and $r$ is the displacement from the fiber core axis. The geometry and RI profiles of the bending MZI along $+\mathrm{Y}\left(0^{\circ}\right)$ and $+\mathrm{Z}\left(90^{\circ}\right)$ axis direction are shown in Fig. 6. So the refractive index difference of our MZI could be approximately modified as

$$
\begin{aligned}
\Delta n(+y) & =n_{1}(1+y C)-n_{2}[1+(-y) C] \\
& =\left(n_{1}-n_{2}\right)+\left(n_{1}+n_{2}\right) y C \\
\Delta n(-y) & =\left(n_{1}-n_{2}\right)+\left(n_{1}+n_{2}\right)(-y) C \\
\Delta n(+z) & =n_{1}(1+z C)-n_{2}(1+z C) \\
& =\left(n_{1}-n_{2}\right)+\left(n_{1}-n_{2}\right) z C \\
\Delta n(-z) & =\left(n_{1}-n_{2}\right)+\left(n_{1}-n_{2}\right)(-z) C
\end{aligned}
$$

Where $n_{1}$ is the refractive index of PRIMZ; $n_{2}$ is the refractive index of un-modified core. From Eq. (5) or Fig. 6(c), it is clear that bending of the MZI in the $+y$ direction $\left(0^{\circ}\right.$ orientation) will result in an increase of $\Delta n(+y)$, and consequently, the effective refractive index difference of the interfering modes, hence, $\Delta n_{\text {eff }}(+y)$ will increase. Thus, according to Eq. (2), the wavelength of the intensity $\operatorname{dip} \lambda_{m}(+y)$ will increase, which means the interference pattern will shift to longer wavelengths i.e. "red" shift with $y>0$. When the bending is in the $-y$ direction (above mentioned $180^{\circ}$ orientation), the opposite effect can be expected. While in the $\pm z$ directions (i.e. $90^{\circ}$ and $270^{\circ}$ orientation), from Eq. (6) or Fig. $6(\mathrm{~d}), \Delta n( \pm z)$ is changed very little with curvature $C$. Hence why the $90^{\circ}$ and $270^{\circ}$ orientations have almost no bend sensitivity. 
Previous work has shown that the device is insensitive to the surrounding refractive index [19]. In addition, the response of the MZI to temperature and axial strain were also investigated.

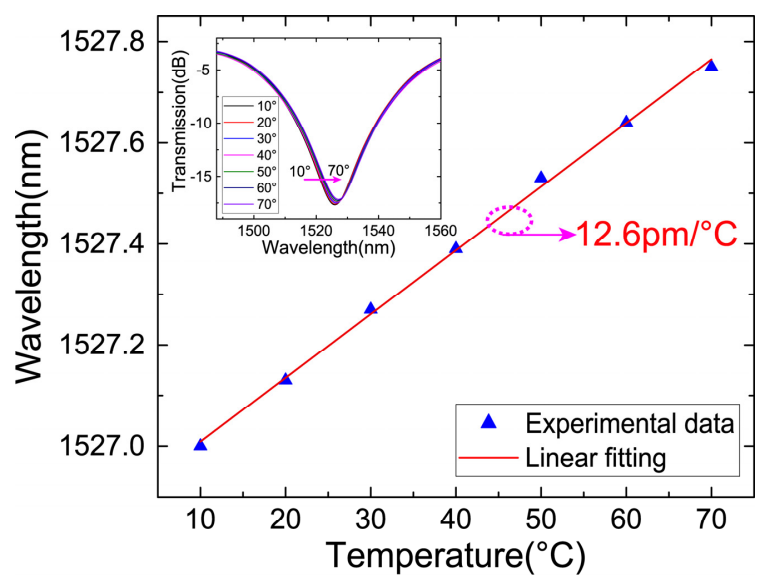

Fig. 7. Temperature response of the device.

To evaluate the temperature response, the device was placed on a heater with the temperature changing from 10 to $70^{\circ} \mathrm{C}$. The shift of the resonance wavelengths versus temperature was tracked. A red shift can be observed when temperature is increasing, as shown in Fig. 7 with a sensitivity of $12.6 \mathrm{pm} /{ }^{\circ} \mathrm{C}$. This corresponds to a crosstalk from temperature for the bend measurements of $4.98 \times 10^{-3} \mathrm{~m}^{-1} /{ }^{\circ} \mathrm{C}$ and $5.63 \times 10^{-3} \mathrm{~m}^{-1} /{ }^{\circ} \mathrm{C}$ at $0^{\circ}$ and $180^{\circ}$ bending orientations, respectively. Therefore, the crosstalk of temperature is so small that it can be neglected in room temperature measurements.

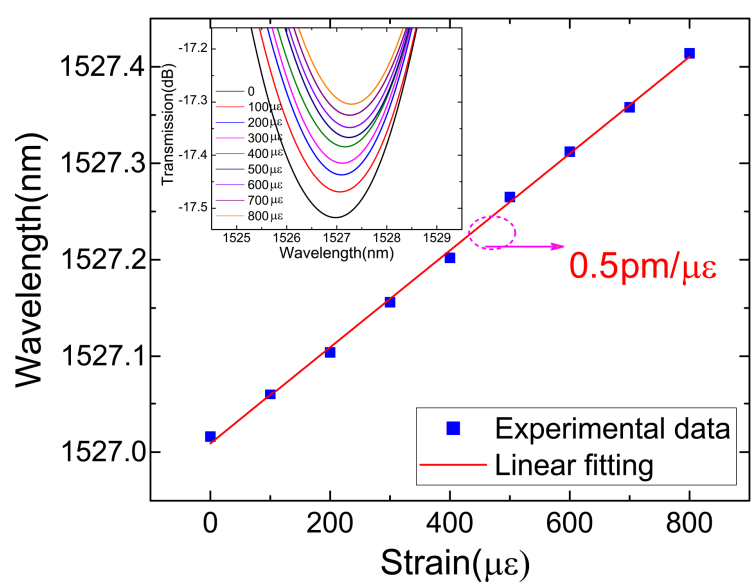

Fig. 8. Axial strain responses of the device.

The strain characteristics of the device were tested by stretching the fiber with the two translation stages. A small redshift can be observed with the increase of strain, as shown in Fig. 8. A linear fit to the experimental data gives a strain sensitivity of $0.5 \mathrm{pm} / \mu \varepsilon$. Such a low strain sensitivity means the proposed device fairly insensitive to the axial strain.

In summary, a compact assembly-free vector bend fiber sensor based on directing fs-laser writing has been demonstrated. The in- fiber-core PRIMZ induced by the fs-laser changes the original SMF to a few-mode fiber (FMF) along the length of the inscription. Thus, the sensor can be regarded as an "SMF-FMF-SMF" sandwich interference structure. Bending the sensor induces a wavelength shift of the interference spectrum. The main features of the proposed sensor include: (i) low cost and simple fabrication since only SMF is needed and the production process takes only a few minutes; (ii) compactness, since the device is only a few millimeters in length; (iii) robustness and assembly-free, there is no need to remove fiber parts, nor the need to splice different fibers together; (iv) high bend sensitivity in a wide curvature range, the device exhibits high sensitivity of 2.53 and $2.24 \mathrm{~nm} / \mathrm{m}^{-1}$ for the $0^{\circ}$ and $180^{\circ}$ orientations in a wide bending range $\left[0-4 \mathrm{~m}^{-1}\right]$, respectively; (v) immunity to SRI, and low temperature crosstalk. These advantages mean that the device is well suited to practical structural monitoring applications.

Funding. National Natural Science Foundation of China (NSFC) (61775074); National 1000 Young Talents Program, China; 111 Project (No. B07038); Natural Science Foundation of Guangdong Province, China (2015A030313633)

Acknowledgment. The authors thank Prof. M. Sumetsky from the Aston Institute of Photonic Technologies for his useful discussion.

\section{References}

1. D. Zhao, X. Chen, K. Zhou, L. Zhang, I. Bennion, W. N. MacPherson, J. S. Barton, and J. D. Jones, Appl. Opt 43, 5425 (2004).

2. G. Mao, T. Yuan, C. Guan, J. Yang, L. Chen, Z. Zhu, J. Shi, and L. Yuan, Opt. Express 25, 144 (2017).

3. T. Yuan, X. Zhong, C. Guan, J. Fu, J. Yang, J. Shi, and L. Yuan, Opt. Express 23, 33378 (2015).

4. G. M. Flockhart, W. N. MacPherson, J. S. Barton, J. D. Jones, L. Zhang, and I. Bennion, Opt. Lett. 28, 387 (2003).

5. P. Saffari, T. Allsop, A. Adebayo, D. Webb, R. Haynes, and M. M. Roth, Opt. Lett. 39, 3508 (2014).

6. T. Allsop, K. Kalli, K. Zhou, Y. Lai, G. Smith, M. Dubov, D. J. Webb, and I. Bennion, Opt. Commun. 281, 5092 (2008).

7. J. Long, J. Wei, and J. Jian, J. Lightwave Technol. 27, 4884 (2009).

8. D. Feng, X. Qiao, and J. Albert, Opt. Lett. 41, 1201 (2016).

9. Q. Zhou, W. Zhang, L. Chen, Z. Bai, L. Zhang, L. Wang, B. Wang, and T. Yan, IEEE Photon. Technol. Lett. 27, 713 (2015).

10. W. Bao, X. Qiao, Q. Rong, N. Hu, H. Yang, Z. Feng, and M. Hu, IEEE Photon. Technol. Lett. 27, 709 (2015).

11. A. V. Newkirk, J. E. Antonio-Lopez, A. Velazquez-Benitez, J. Albert, R. Amezcua-Correa, and A. Schulzgen, Opt. Lett. 40, 5188 (2015).

12. J. Villatoro, A. Van Newkirk, E. Antonio-Lopez, J. Zubia, A. Schulzgen, and R. Amezcua-Correa, Opt. Lett. 41, 832 (2016).

13. S. Wang, Y.-X. Zhang, W.-G. Zhang, P.-C. Geng, T.-Y. Yan, L. Chen, Y.-P. Li, and W. Hu, IEEE Photon. Technol. Lett. 29, 822 (2017).

14. Z. Ou, Y. Yu, P. Yan, J. Wang, Q. Huang, X. Chen, C. Du, and H. Wei, Opt. Express 21, 23812-23821 (2013).

15. J. Villatoro, V. P. Minkovich, and J. Zubia, Opt. Lett. 40, 3113 (2015).

16. L. Zhang, W. Zhang, L. Chen, T. Yan, L. Wang, B. Wang, and Q. Zhou, IEEE Photon. Technol. Lett., 1 (2015).

17. Q. Huang, Y. Yu, X. Li, X. Chen, Y. Zhang, W. Zhou, and C. Du, Opt. Express 23, 3010 (2015).

18. S. Zhang, W. Zhang, S. Gao, P. Geng, and X. Xue, Opt. Lett. 37, 4480 (2012).

19. P. Chen, X. Shu, and K. Sugden, Optics Letters 42, 4059 (2017).

20. K. Nagano, S. Kawakami, and S. Nishida, Appl. Opt 17, 2080 (1978). 


\section{Full References}

1. D. Zhao, X. Chen, K. Zhou, L. Zhang, I. Bennion, W. N. MacPherson, J. S. Barton, and J. D. Jones, "Bend sensors with direction recognition based on long-period gratings written in D-shaped fiber," Appl. Opt 43, 54255428 (2004).

2. G. Mao, T. Yuan, C. Guan, J. Yang, L. Chen, Z. Zhu, J. Shi, and L. Yuan, "Fiber Bragg grating sensors in hollow single- and two-core eccentric fibers," Opt. Express 25, 144-150 (2017).

3. T. Yuan, X. Zhong, C. Guan, J. Fu, J. Yang, J. Shi, and L. Yuan, "Long period fiber grating in two-core hollow eccentric fiber," Opt. Express 23, 33378-33385 (2015).

4. G. M. Flockhart, W. N. MacPherson, J. S. Barton, J. D. Jones, L. Zhang, and I. Bennion, "Two-axis bend measurement with Bragg gratings in multicore optical fiber," Opt. Lett. 28, 387-389 (2003).

5. P. Saffari, T. Allsop, A. Adebayo, D. Webb, R. Haynes, and M. M. Roth, "Long period grating in multicore optical fiber: an ultra-sensitive vector bending sensor for low curvatures," Opt. Lett. 39, 3508-3511 (2014).

6. T. Allsop, K. Kalli, K. Zhou, Y. Lai, G. Smith, M. Dubov, D. J. Webb, and I. Bennion, "Long period gratings written into a photonic crystal fibre by a femtosecond laser as directional bend sensors," Opt. Commun. 281, 5092-5096 (2008).

7. J. Long, J. Wei, and J. Jian, "Directional Bend Sensing With a CO2-LaserInscribed Long Period Grating in a Photonic Crystal Fiber," J. Lightwave Technol. 27, 4884-4891 (2009).

8. D. Feng, X. Qiao, and J. Albert, "Off-axis ultraviolet-written fiber Bragg gratings for directional bending measurements," Opt. Lett. 41, 12011204 (2016).

9. Q. Zhou, W. Zhang, L. Chen, Z. Bai, L. Zhang, L. Wang, B. Wang, and T. Yan, "Bending Vector Sensor Based on a Sector-Shaped Long-Period Grating," IEEE Photon. Technol. Lett. 27, 713-716 (2015).

10. W. Bao, X. Qiao, Q. Rong, N. Hu, H. Yang, Z. Feng, and M. Hu, "Sensing Characteristics for a Fiber Bragg Grating Inscribed Over a Fiber Core and Cladding," IEEE Photon. Technol. Lett. 27, 709-712 (2015).

11. A. V. Newkirk, J. E. Antonio-Lopez, A. Velazquez-Benitez, J. Albert, R. Amezcua-Correa, and A. Schulzgen, "Bending sensor combining multicore fiber with a mode-selective photonic lantern," Opt. Lett. 40, 5188-5191 (2015).

12. J. Villatoro, A. Van Newkirk, E. Antonio-Lopez, J. Zubia, A. Schulzgen, and R. Amezcua-Correa, "Ultrasensitive vector bending sensor based on multicore optical fiber," Opt. Lett. 41, 832-835 (2016).

13. S. Wang, Y.-X. Zhang, W.-G. Zhang, P.-C. Geng, T.-Y. Yan, L. Chen, Y.-P. $\mathrm{Li}$, and W. Hu, "Two-Dimensional Bending Vector Sensor Based on the Multimode-3-Core-Multimode Fiber Structure," IEEE Photon. Technol. Lett. 29, 822-825 (2017).

14. Z. Ou, Y. Yu, P. Yan, J. Wang, Q. Huang, X. Chen, C. Du, and H. Wei, "Ambient refractive index-independent bending vector sensor based on seven-core photonic crystal fiber using lateral offset splicing," Opt. Express 21, 23812-23821 (2013)

15. J. Villatoro, V. P. Minkovich, and J. Zubia, "Photonic crystal fiber interferometric vector bending sensor," Opt. Lett. 40, 3113-3116 (2015).

16. L. Zhang, W. Zhang, L. Chen, T. Yan, L. Wang, B. Wang, and Q. Zhou, "A Fiber Bending Vector Sensor Based on M-Z Interferometer Exploiting Two Hump-Shaped Tapers," IEEE Photon. Technol. Lett., 1-1 (2015).

17. Q. Huang, Y. Yu, X. Li, X. Chen, Y. Zhang, W. Zhou, and C. Du, "Microbending vector sensor based on six-air-hole grapefruit microstructure fiber using lateral offset splicing," Opt. Express 23, 3010-3019 (2015).

18. S. Zhang, W. Zhang, S. Gao, P. Geng, and X. Xue, "Fiber-optic bending vector sensor based on Mach-Zehnder interferometer exploiting lateral-offset and up-taper," Opt. Lett. 37, 4480-4482 (2012).

19. P. Chen, X. Shu, and K. Sugden, "Ultra-compact all-in-fiber-core MachZehnder interferometer," Opt. Lett. 42, 4059-4062 (2017).

20. K. Nagano, S. Kawakami, and S. Nishida, "Change of the refractive index in an optical fiber due to external forces," Appl. Opt 17, 20802085 (1978). 